\section{MOLECULAR DYNAMICS SIMULATION OF DIFFUSE} SCATTERING IN QUARTZ

K. Kihara N. Matsui

Kanazawa University Department of Earth Sciences Kakuma KANAZAWA 920-1192 JAPAN

X-ray diffuse scattering in quartz along non-radial streaks joining symmetryequivalent points of index 400 (H. Arnold, Zeit. Krist. (1965) 121, 145 etc), was investigated by molecular dynamics (MD) simulation in the NPT ensemble. The MD was conducted at 14 points of temperature for the 2400 hexagonal cells using the semi-empirical energy parameters of G. Kramer et al. (Phys. Rev. (B) (1991) 43, 5068). In this MD system, atoms show two characteristic motions: one is with smaller amplitudes in the a1 (or a2) sites and the other with larger amplitudes over the a1 and a 2 sites. The correlation length in the latter motions grows to a maximum in the vicinity of the transition point. X-ray intensities calculated for all atoms in 4096 steps, each separated by $10 \mathrm{fs}$, showed pronounced non-radial diffuse streaks, even far below the transition point, with wave vector qfs in the $\gamma$-M directions. The temperature dependence of the intensities is in harmony with the measurement of $\mathrm{H}$. Arnold, and is nearly paralleled by that of the a 1-a 2 transferring frequency of atoms, convincing us that the diffuse scattering arises, not from the simple rotational motions of corner-linked tetrahedra around the a1- or a2-orientation, but from the transferring motions in collected modes with non-zero qfs in $\gamma-\mathrm{M}$. The atomic view of the lowest soft acoustic branches in $\gamma-\mathrm{M}$ in $\alpha$-quartz are depicted by the collected modes of a1-a2 transferring motions, which are activated by the rotational modes in the A1 symmetry.

\section{Keywords: QUARTZ DIFFUSE SCATTERING MOLECULAR DYNAMICS SIMULATION}

\section{Acta Cryst. (2002). A58 (Supplement), C269}

\section{MOLECULAR DYNAMICS SIMULATIONS OF $\mathrm{LiMn}_{2} \mathrm{O}_{4}$ SPINEL}

K. Tateishi D. J. du Boulay N. Ishizawa

Tokyo Institute of Technology Materials and Structures Laboratory 4259

Nagatsuta, Midori YOKOHAMA 226-8503 JAPAN

Molecular dynamics simulations of $\mathrm{LiMn}_{2} \mathrm{O}_{4}$ were carried out to validate the charge density study of $\mathrm{LiMg}_{1 / 6} \mathrm{Mn}_{11 / 6} \mathrm{O}_{4}$ spinel experimentally obtained in our study. Prior to this it had been thought that the Li ion moved from the ideal 8a site to the neighboring empty $16 \mathrm{c}$ site of the $F d-3 m$. However our MD calculations have suggested that it moves from the positions $0.10 \AA$ apart from 8a to the positions $0.38 \AA$ apart from $16 \mathrm{c}$ and there is a smaller probability that Li ions are actually located at the ideal $8 \mathrm{a}$ or $16 \mathrm{c}$ sites. It was initially thought that such lithium ion migration was unlikely to take place in the ideal spinel structure and actually with the assumption of all equivalent $\mathrm{Mn}^{3.5+}$ ions our MD calculations reproduced the ideal spinel structure and showed that $\mathrm{Li}$ ion migration does not take place. In MD calculations with the assumption of $\mathrm{Mn}^{3+}$ and $\mathrm{Mn}^{4+}$ co-existence, there are four possible tetrahedral configurations $\left[\mathrm{OLiM}_{3}\right]^{\mathrm{n}+}\left(\mathrm{M}=\mathrm{Mn}^{3+}\right.$ or $\left.\mathrm{Mn}^{4+} \mathrm{n}=+8,+9,+10,+11\right)$ and the oxygen atoms undergo different displacements in each. Because of these displacements, the $\mathrm{LiO}_{4}$ tetrahedra appear distorted and $\mathrm{Li}$ ions can migrate to neighboring octahedra through the extended tetrahedral face. Experimental data show that $\mathrm{Li}$ ions exist in both tetrahedra and octahedra at room temperature. It is therefore quite likely that distinct $\mathrm{Mn}^{3+}$ and $\mathrm{Mn}^{4+}$ ions coexist in the spinel structure and the transition of electric charges of the Mn ions is primarily responsible for the lithium ion migration, in accordance with our calculations.

Keywords: MOLECULAR DYNAMICS SIMULATION LITHIUM MANGANATE SPINEL LITHIUM ION RECHARGEABLE BATTERY
Acta Cryst. (2002). A58 (Supplement), C269

\section{ELECTRONIC STRUCTURE CALCULATION OF $\beta-\mathrm{MnO}_{2} \mathrm{HAVING}^{-}$} IMPURITIES AND DEFECTS

D.Y. Lee B.S. Kim J.S. Song

Korea Electrotechnology Research Institute Electric and Magnetic Devices Research Group Sungju-Dong 28-1 CHANGWON KYUNGNAM 641-120 SOUTH KOREA

The electronic structure of $\beta-\mathrm{MnO}_{2}$ was calculated with the Discrete Variation $\mathrm{X}-\alpha(\mathrm{DV}-\mathrm{X}-\alpha)$ method based on the density functional theory. The theoretical $a b$-initio electronic structure calculation is one of useful tools used for material design to elucidate the relationship between a crystal structure and its properties. The experimental diffraction method has some limits on the determination of electronic charge density distribution in crystals having defects and impurities. DV-X- $\alpha$ method overcomes these limits and compensates the results of experimental methods. DV-X- $\alpha$ method uses a cluster having below 100 atoms modeled on the experimentally determined crystal structure. It is possible to calculate the electronic structure of crystals having vacancies, impurities in substitutive and interstitial sites, interfaces and surfaces with this method. $\beta-\mathrm{MnO}_{2}$ has been used for a catalyst and an electrode, because of good electric conductivity and oxygen-generating ability. In this research the effect of impurities and vacancies on the electronic structure of $\beta-\mathrm{MnO}_{2}$ were investigated with DV-X- $\alpha$ method in order to obtain basic data for designing new electrode materials. Clusters used for this purpose were $\mathrm{Mn}_{3} \mathrm{O}_{15}, \mathrm{Mn}_{11} \mathrm{O}_{44}$ and $\mathrm{Mn}_{15} \mathrm{O}_{30}$. Central $\mathrm{Mn}$ atom in Mn-centered clusters was substituted to transition metals in order to investigate the effect of cation impurities. Oxygen atom in the center position of an oxygen-centered cluster was eliminated for investigating vacancy effect. The results obtained from calculations include the charge density, the spin density, the density of state, net charge transfer, the bond overlap population and the wave function.

\section{Keywords: $\mathrm{MNO}_{2}$ ELECTRONIC STRUCTURE DENSITY FUNCTIONAL THEORY}

\section{Acta Cryst. (2002). A58 (Supplement), C269 \\ CONFORMATIONAL ANALYSIS OF ELASTIC REPEAT MOTIF IN TITIN}

M. Sun Y.F. Zhao

Institute of Chemical and Crystallography Tianjin Normal University HeXi Distinct WeiJing Road 241 Tianjin Normal University TIANJIN 300074 CHINA

The extension of PEVK segment of elastic protein titin is a key event on the elastic response of striated muscle to passive stretch. In this article, tetrapeptides KVPE and EVPK in either phosphorylated and unphosphorylated forms have been designed to mimic the elastic repeat motif in PEVK according to the reported statistic sequence analysis. The conformational analysis was carried out with Monte Carlo Multiple Minimum (MCMM) search and Monte Carlo/Stochastic Dynamics(MC/SD) simulation in order to understand what are the preferred conformations and how the conformations were regulated by phosphorylation. All calculations are performed with MacroModel version 7.0 on SGI O2 R10000 workstation. The sampled multiple conformations show a large range of elasticity with end-to-end distances d range from 4 to $10 \AA$, and there are obviously two peaks in each d-conformational distribution plot with distance $\mathrm{d}$ around 5.5 and $9 \AA$ A, respectively. Conformations with $\mathrm{d}$ around 5.5 and $9 \AA$ are significantly populated and compose two clusters of preferred conformations with different end-to-end distances. This conformational property may be the molecular basic for the elasticity of PEVK. In addition, the two conformation clusters are correspondent with cis or trans geometry of amide bond of $\mathrm{P}$, therefore the cis trans isomerization of proline may work as a key effect in regulating the elasticity. The located low energy conformations from MCMM also indicate that the phosphorylation of specific amino acid induces a large orientation changes of Lys or Glu side chain and this may also be a possible factor affecting muscle elasticity.

\section{Keywords: CONFORMATIONAL ANALYSIS ELASTIC REPEAT MOTIF PEVK TITIN}

